Polish Journal of Microbiology

2011, Vol. 60, No 2, 163-168

ORGINAL PAPER

\title{
Association between Existence of Integrons and Multi-Drug Resistance in Acinetobacter Isolated from Patients in Southern Iran
}

\author{
SARA JAPONI ${ }^{1}$, AZIZ JAPONI ${ }^{2 *}$, SHOREH FARSHAD ${ }^{2}$, AHYA ABDI ALI ${ }^{1}$ \\ and MARZIEH JAMALIDOUST ${ }^{2}$
}

${ }^{1}$ Department of Biology, Alzahra University, Tehran, Iran

${ }^{2}$ Alborzi Clinical Microbiology Research Center, Nemazee hospital, Shiraz, Iran

Received 2 September 2010, revised 5 January 2011, accepted 20 January 2011

Abstract

Nosocomial infections caused by multi-drug resistant Acinetobacter pose a serious problem in many countries. This study aimed at determining the antibiotic susceptibility patterns and prevalence of different classes of integrons in isolated Acinetobacter. In addition, the association between production of specific bands in PCR assay and magnitude of multi-drug resistance was investigated. In total, 88 Acinetobacter strains were isolated from patients from October 2008 through September 2009. The Minimal inhibitory concentration (MIC) of 12 antibiotics conventionally used in clinics against the isolates, was determined by E-test method. The existence of integron classes was investigated by PCR assay through the amplification of integrase genes. The most effective antibiotic against Acinetobacter was colistin with $97.7 \%$ activity, followed by imipenem (77.3\%) and meropenem (72.7\%). The presence of integron classes 1 and 2 in 47 (53.4\%) isolates was confirme, However, no class 3 was detected. The proportion of class 1, compared with class 2 , was high (47.7\% vs. 3.4\%). The association between multi-drug resistance to norfloxacin, ceftazidime, gentamicin, ciprofloxacin, cefepime and amikacin and the presence of integrons was statistically significant. However, the association was not remarkable in many of the isolates which exhibited resistance to the rest of antibiotics. This may imply that in addition to integrons, other resistance determinants such as transposon and plasmid may also contribute to resistance. To reduce the pressure on sensitive isolates, comprehensive control measures should be implemented. Furthermore, wise application of effective antibiotics could help alleviate the situation. Colistin is the most effective antibiotic in vitro against Acinetobacter.

Ke y words: Acinetobacter, integrons, multi-drug resistance, PCR assay

\section{Introduction}

Acinetobacter is a gram-negative bacterium, which is a very effective human colonizer found in many health care environments (Bergogne-Berezin and Towner, 1996; Perez et al., 2007). The combination of its environmental resilience and its wide range of resistance determinants renders it a successful nosocomial pathogen (Nordmann, 2004). Nowadays, A. baumannii is emerging as a cause of numerous global outbreaks, displaying ever-increasing rates of resistance (Villegas and Hartstein, 2003).

Although six classes of integrons exist (Neild et al., 2001), three main classes have been described (RoweMagnus and Mazel, 1999). All integrons have a 5' conserved segment, including an intI gene encoding an integrase and an attI recombination site, but have distinct 3' conserved segments. As for the class 1 integrons, the 3 ' conserved segment includes three open reading frames (ORFs): qacE $\Delta 1$, a deletion derivative of the antiseptic resistance gene qacE; the sull sulfonamide resistance gene; and ORF5, of unknown function (Radström et al., 1994). The second class of integrons was found in transposon Tn7 and its derivatives, and its 3' conserved segment contains five ths genes involved in the movement of the transposon. A single class 3 integron has been reported to date, but its 3' conserved segment has not been characterized (Arakawa et al., 1995). Class 1 integron is more frequent in Acinetobacter species (Koeleman et al., 2000; Galleco and Towner, 2001; Gaur et al., 2006; Ploy et al., 2000; Xu et al., 2008).

The objectives of this study were to determine the prevalence of different classes of integrons in isolated Acinetobacter and to find out the association between the presence of integrons and antibiotics resistance. Furthermore, the relationship between the productions of specific bands in PCR assay with the extension of multi-drug resistance was studied.

* Corresponding author: A. Japoni, Alborzi Clinical Microbiology Research Center, Nemazee hospital, Shiraz University of Medical Sciences, post code: 71037-11351, Shiraz, Iran; phone: +98-711-6474264; fax: +98-711- 6474303; e-mail: Japonia@hotmail.com 


\section{Experimental}

\section{Material and Methods}

Isolation of Acinetobacter. Eighty eight Acinetobacter strains were isolated from patients hospitalized in Nemazee hospital, affiliated with Shiraz University of Medical Sciences, Iran during the period from October 2008 to September 2009. Identification of the isolates was carried out using the API 20E system (bioMérieux, Marcy l'Etoile, France).

MIC determination. Minimal inhibitory concentrations (MICs) of 12 antibiotics including, ciprofloxacin, colistin, ceftazidime, ampicillin/sulbactam, imipenem, meropenem, gentamicin, norfloxacin, amikacin, cefepeme, tobramycin and cefoperazon/sulbactom against the isolates were determined by the E-test method and the results were interpreted as recommended by the manufacturer's instructions.

DNA extraction and PCR assay. Bacteria DNA were harvested by conventional phenol-chloroform extraction method. The quantity of DNA was determined by Nanodrop (NanoDrop Technologies, Wilmington, Delaware USA) and adjusted $50 \mathrm{ng} \mu^{-1}$. Determination of integron classes was performed by multiplex PCR using the primers described in Table I. The primers were obtained from TIB MOLBIOL Syntheselabor $\mathrm{GmbH}$ (Berlin, Germany). PCR assay was performed in $20 \mu \mathrm{l}$ volume, containing $0.4 \mathrm{mM}$ deoxynucleoside triphosphate (dNTP), $2 \mathrm{ml}$ of 10X PCR buffer, $1 \mathrm{U}$ of Taq polymerase (Fermentas, Lithuania), $0.6 \mathrm{mM} \mathrm{MgCl}_{2}$, $0.25 \mathrm{mM}$ of each primer and $250 \mathrm{ng}$ DNA in $5 \mu \mathrm{l} \mathrm{vol-}$ ume were added to the reaction mixtures. PCR was performed under pre-denaturation at $94^{\circ} \mathrm{C}$ for $5 \mathrm{~min}$ utes, followed by 35 cycles of $94^{\circ} \mathrm{C}$ for 30 seconds, $55^{\circ} \mathrm{C}$ for 30 seconds, and $72^{\circ} \mathrm{C}$ for 30 seconds ending with a final extension step at $72^{\circ} \mathrm{C}$ for $5 \mathrm{~min}$ and held at $8^{\circ} \mathrm{C}$. Products were electrophoresed in $2 \%$ agarose, stained by ethidium bromide and video images were obtained by gel documentation (Uvtec, Sigma, Germany) system.

Primers Int $1 F$ and Int $1 R$ were used to amplify a 160 bp fragment of the intI1 gene for the class 1 integrase and the primers $I n t 2 F$ and $I n t 2 R$ amplified a fragment of $288 \mathrm{bp}$, specific for the intI2 gene. Primers Int $3 F$ and Int $3 R$ were used to amplify a specific int I3 gene.

Detection of the complete gene composition of class 1 integrons was performed with primers for the $5^{\prime}$ and 3' conserved segments. This PCR also permitted the determination of the size of any inserted gene cassette. The size range of the detected inserted gene cassette varied from 500 to $>3000 \mathrm{bp}$.

PCR assay to detect complete gene makeup of class 1 integron was carried out in $20 \mu$ volume with the same concentration mixture mentioned above. The only modification was increased to $3 \mathrm{U}$ amount of Smart Taq polymerase (Fermentas, Lithuania). PCR amplification program was as follows: 5 minutes of initial denaturation at $94^{\circ} \mathrm{C}, 1 \mathrm{~min}$ of denaturation at $94^{\circ} \mathrm{C}, 1 \mathrm{~min}$ of annealing at $55^{\circ} \mathrm{C}$, and 30 seconds of extension at $72^{\circ} \mathrm{C}$ for a total of 35 cycles. Five seconds were added to the extension time at each cycle.

Statistical analysis. Correlation between antibiotic resistance patterns and presence of different classes of integron was determined by Chi-square and Fisher's Exact test by SPSS version 15 software. The significant level was defined as $\mathrm{P}<0.05$.

\section{Results}

Acinetobacter spp was mostly isolated from the blood, $35(39.8 \%)$ and predominantly in men (70\%), than in women (30\%). A. baumannii was dominant in the isolated bacteria 79 (89.8\%), followed by Acinetobacter lwoffii 8 (9.1\%). $97.7 \%$ of Acinetobacter isolates were susceptible to colistin, $77.3 \%$ to imipenem, $72.7 \%$ to meropenem, $67 \%$ to cefoperazon/sulbactam, $63.6 \%$ to tobramycin, $61.4 \%$ to ampicillin/sulbactam, $26.1 \%$ to ciprofloxacin, $25 \%$ to amikacin, $23.8 \%$ to norfloxacin, $20.4 \%$ to gentamicin, $19.3 \%$ to cefepime and $18.2 \%$ to ceftazidime.

Diagnosis of integrons was carried out by multiplex PCR assay. The presence of intI1 and intI2 was confirmed while intI3 was not detected. Figure 1 demonstrates the presence of integrase 1 and 2. In total,

Table I

Primers used in this study

\begin{tabular}{|c|c|c|}
\hline Primer & Nucleotide sequence & References \\
\hline Int1-F & 5' CAG TGG ACA TAA GCC TGT TC 3' & \multirow{4}{*}{ Koeleman et al., 2000} \\
\hline Int $1-R$ & 5’ CCC GAG GCA TAG ACT GTA 3’ & \\
\hline Int $2-F$ & 5' TTG CGA GTA TCC ATA ACC TG 3’ & \\
\hline Int2-R & 5’ TTA CCT GCA CTG GAT TAA GC 3’ & \\
\hline Int3-F & 5’ ACG GAT CTG CCA AAC CTG ACT 3’ & \multirow{2}{*}{ Ploy et al., 2000} \\
\hline Int $3-R$ & 5’ GCC TCC GGC AGC GAC TTT CAG 3' & \\
\hline CS-F & 5’ GGC ATC CAA GCA GCA AG 3’ & \multirow{2}{*}{ Lévesque et al., 1995} \\
\hline$C S-R$ & 5’ AAG CAG ACT TGA CCT GA 3' & \\
\hline
\end{tabular}


Table II

Prevalence of different classes of integrons in Acinetobacter isolates

\begin{tabular}{|c|c|l|}
\hline Percentage & Number & \multicolumn{1}{|c|}{ Integron } \\
\hline 47.7 & 42 & Class I \\
\hline 3.4 & 3 & Class II \\
\hline 2.3 & 2 & Class I \& Class II \\
\hline 0 & 0 & Class III \\
\hline 46.6 & 41 & Without integron \\
\hline 100 & 88 & Total \\
\hline
\end{tabular}

47 (53.4\%) Acinetobacter strains exhibited either a class 1 integrase or class 2 integrase or both of them. Table II shows the prevalence of different classes of integron in Acinetobacter isolates.
Forty five (57\%) of the 79 A.baumannii strains, and $2(25 \%)$ of the 8 A. lwoffii strains carry an integron. The frequencies of the different antibiotic resistance patterns and their association with integrons were assessed. Based on this evaluation, strains exhibiting resistance to the panel of antibiotics including norfloxacin, ceftazidime, gentamicin, ciprofloxacin, amikacin, cefepime and norfloxacin, ceftazidime, gentamicin, ciprofloxacin, ampicillin/sulbactam, amikacin, cefoperazon/sulbactom, cefepime showed high prevalence of class 1 integrons.

Data also indicate an association between the panel of antibiotics including norfloxacin, ceftazidime, gentamicin, ciprofloxacin, amikacin, tobramycin, cefepime and class 2 integron (Table III). Amplification of integron class 1 produced bands ranging between 500 to

Table III

Association between Antibiotic resistance and integron classes

\begin{tabular}{|c|c|c|c|c|}
\hline \multirow{2}{*}{ Integron } & \multicolumn{4}{|c|}{ Antibiotic resistance pattern } \\
\hline & Total & Class I \& II & Class II & Class I \\
\hline $\mathrm{TZ}$ & 1 & - & - & 1 \\
\hline TZ-AK & - & - & 1 & 1 \\
\hline AB-PM & - & - & - & 1 \\
\hline AK-TM & - & - & - & - \\
\hline GM-TM & - & - & - & 1 \\
\hline AB-IP-MP & - & - & - & 1 \\
\hline NX-TZ-GM-TM-PM & - & - & - & 1 \\
\hline TZ-GM-AK-TM-PM & 1 & - & - & 1 \\
\hline NX-TZ-GM-CI-AK-PM & 11 & - & - & 13 \\
\hline NX-TZ-GM-CI-AB-PM & 2 & - & - & 2 \\
\hline NX-TZ-GM-AK-TM-PM & - & - & - & 1 \\
\hline NX-TZ-CI-AK-MP-PM & - & - & - & 1 \\
\hline NX-TZ-GM-CI-AK-CPS-PM & 1 & - & - & 2 \\
\hline NX-TZ-GM-CI-AK-TM-PM & 4 & 3 & - & 14 \\
\hline NX-TZ-GM-CI-AK-PM-CO & - & - & - & 1 \\
\hline NX-TZ-GM-AK-IP-TM-MP-PM & 1 & - & - & 1 \\
\hline NX-TZ-GM-CI-AB-AK-CPS-PM & 8 & - & - & 11 \\
\hline NX-TZ-GM-CI-AK-TM-MP-PM & 1 & - & - & 2 \\
\hline NX-TZ-GM-CI-AB-AK-TM-PM & 1 & - & - & 1 \\
\hline TZ-GM-AB-IP-TM-MP-PM-CO & - & - & - & 1 \\
\hline TZ-GM-CI-AK-IP-TM-MP-PM & 1 & - & - & 1 \\
\hline NX-TZ-GM-CI-AB-AK-TM-MP & 1 & - & - & 1 \\
\hline NX-TZ-GM-CI-AB-IP-CPS-MP-PM & 2 & - & - & 2 \\
\hline NX-TZ-GM-CI-AB-AK-IP-CPS-MP-PM & 5 & - & 1 & 6 \\
\hline NX-TZ-GM-CI-AB-AK-IP-TM-CPS-MP-PM & 1 & - & - & 8 \\
\hline Sensitive & 1 & - & - & 12 \\
\hline Total & 42 & 3 & 2 & 88 \\
\hline
\end{tabular}

Abbreviations: CI - ciprofloxacin; CO - colistin; TZ - ceftazidime; AB - ampicillin/sulbactam; IP - imipenem; MP - meropenem; GM - gentamicin; NX-norfloxacin; AK - amikacin; PM - cefepime; TM - tobramycin; CPS - cefoperazon/sulbactom. 
Table IV

Size of amplicons when primers used to amplify integron class 1

\begin{tabular}{|l|c|}
\hline \multicolumn{1}{|c|}{ Pattern of integron I bands $(\mathrm{pb})$} & $\begin{array}{c}\text { No. of } \\
\text { isolates }\end{array}$ \\
\hline $500,600,1300$ & 1 \\
\hline $500,800,1200$ & 1 \\
\hline $500,600,800,1200$ & 13 \\
\hline $500,600,800,1200,2500$ & 5 \\
\hline $500,600,800,1200,>3000$ & 1 \\
\hline $500,800,900,1200,2500$ & 1 \\
\hline $500,800,1000,1200,2500$ & 2 \\
\hline $620,900,1300,1700,>3000$ & 1 \\
\hline $500,600,800,900,1200,2500$ & 2 \\
\hline $500,600,800,1000,2300,2500$ & 1 \\
\hline $500,600,800,1200,1500,2500$ & 2 \\
\hline $500,600,800,1200,2500,3000$ & 1 \\
\hline $500,800,1000,1200,1500,2500$ & 4 \\
\hline $500,600,800,900,1200,1500,2500$ & 1 \\
\hline $500,600,800,1000,1200,1500,2500$ & 1 \\
\hline $500,600,800,1200,1500,2400,2500$ & \\
\hline $500,600,800,900,1200,1500,1700,2500,>3000$ & 1 \\
\hline $500,600,750,800,900,1200,1300,1500,2500,>3000$ & 1 \\
\hline
\end{tabular}

$>3000$ bp and the strains containing bands with 500, 600,800 and 1200 bp were more frequent (Table IV).

The association between drug resistance to norfloxacin, ceftazidime, gentamicin, ciprofloxacin, cefepime, amikacin and the presence of integrons was statistically significant, while no association was observed between colistin, imipenem, meropenem, cefoperazon/sulbactom, tobramycin, ampicillin/sulbactam and integron (Table V).

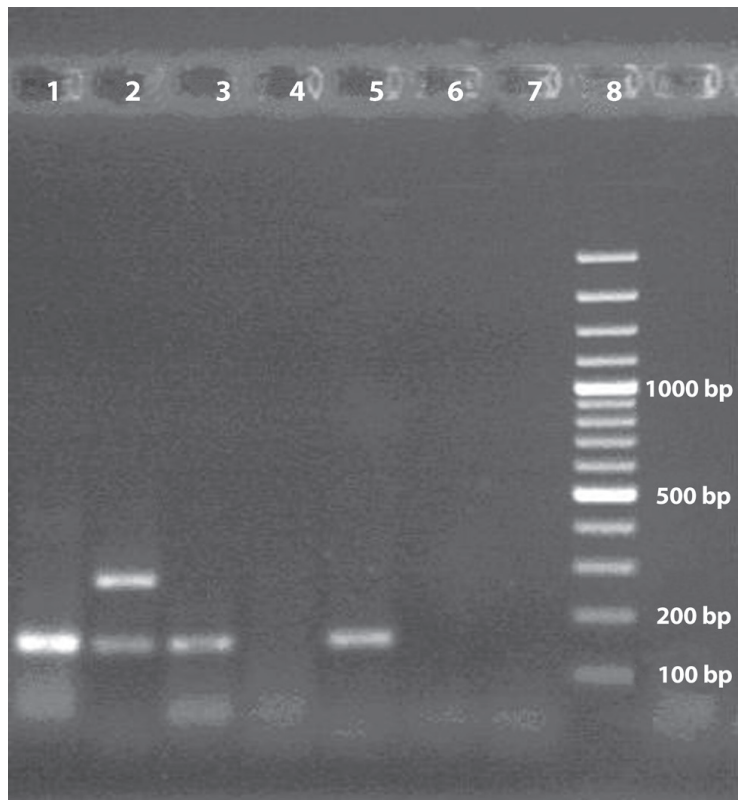

Fig. 1. Detection of integrons by amplification of integrase. Lane 8, 100 bp DNA ladder (MBI Fermentas, Hanover, MD); lanes 1, 3, 5, integrase 1 amplicons (160 bp); lane 2, both integrase 1(160 bp) and integrase 2 amplicons (288 bp). Lane 4, 6 and 7 were integron negative.

\section{Discussion}

Acinetobacter infections are complicated in hospitalized patients due to the acquisition of multi-drug resistance. Most samples in the present study were isolated from the blood (39.8\%). Similar data were obtained previously in the same region (Feizabadi et al., 2008). Dissemination of Acinetobacter through blood may

Table $\mathrm{V}$

Association between the existence of integron and antibiotic resistance in 88 Acinetobacter isolates

\begin{tabular}{|l|c|c|c|c|}
\hline $\begin{array}{c}\text { Association } \\
\text { with integron }\end{array}$ & $\begin{array}{c}\text { \% Resistance } \\
\text { of total (total no) }\end{array}$ & $\begin{array}{c}\text { \% Resistance } \\
\text { int-negative (no) }\end{array}$ & $\begin{array}{c}\text { \% Resistance } \\
\text { int-positive }^{1} \text { (no) }\end{array}$ & Antibiotic \\
\hline Colistin & $0(0)$ & $2.3(2)$ & $2.3(2)$ & 0.126 \\
\hline Imipenem & $13.6(12)$ & $9.1(8)$ & $22.7(20)$ & 0.501 \\
\hline Meropenem & $15.9(14)$ & $11.4(10)$ & $27.3(24)$ & 0.571 \\
\hline Cefoperazone/sulbactam & $21.6(19)$ & $11.4(10)$ & $33(29)$ & 0.110 \\
\hline Tobramycin & $14.8(13)$ & $21.6(19)$ & $36.4(32)$ & 0.069 \\
\hline Ampicillin/sulbactam & $25(22)$ & $13.6(12)$ & $38.6(34)$ & 0.092 \\
\hline Ciprofloxacin & $48.9(43)$ & $25(22)$ & $73.6(65)$ & $\mathbf{P}<\mathbf{0 . 0 5}$ \\
\hline Amikacin & $47.7(42)$ & $27.3(24)$ & $75(66)$ & $\mathbf{0 . 0 0 1}$ \\
\hline Norfloxacin & $48.9(43)$ & $27.3(24)$ & $76.2(67)$ & $\mathbf{P}<\mathbf{0 . 0 5}$ \\
\hline Gentamicin & $51.1(45)$ & $28.4(25)$ & $79.5(70)$ & $\mathbf{P}<\mathbf{0 . 0 5}$ \\
\hline Cefepime & $51.1(45)$ & $29.5(26)$ & $80.6(71)$ & $\mathbf{P}<\mathbf{0 . 0 5}$ \\
\hline Ceftazidime & $52.3(46)$ & $29.5(26)$ & $81.8(72)$ & $\mathbf{P}<\mathbf{0 . 0 5}$ \\
\hline
\end{tabular}

${ }^{1}$ - int-positive: integron positive in multiplex PCR assay; ${ }^{2}$ - int-negative: integron negative in multiplex PCR assay.

${ }^{3}$ - Significant values are in bold. 
indicate the role of the bloodstream in spreading the infection (Gisneous and Rodriguez-Bano, 2002). Consistent with previous studies, A. baumannii is the predominant species in clinical isolates (Seifert et al., 1993; Towner, 2009).

The three most effective antibiotics against Acinetobacter were found to be colistin, imipenem and meropenem. Despite being the most effective antibiotic against Acinetobacter in vitro, colistin use is limited only to life threatening conditions due to its serious side effects (Reed et al., 2001; Lewis and Lewis, 2004). Nevertheless, observation of high resistance rate of Acinetobacter to the majority of the tested antibiotics has limited the use of alternative effective antibiotics. More likely, resistance genes are acquired via genetic elements such as integrons, plasmids and transposons (Perez et al., 2007). In this regard, the role of integron is remarkable due to possessing a strong capturing system (Gonzalez et al., 1998; Seward, 1999; Turton et al., 2005). Continuous capturing of antibiotic resistance genes in Acinetobacter will extend quickly, so with more uncontrolled administration of antibiotics in hospitals and clinics, the possibility of acquiring resistance will be increased. To overcome progressive antibiotic resistance, rational and timely administration of effective antibiotics should be implemented. The present study on the existence of integron revealed that $53.4 \%$ of the isolates contained integron classes 1 or 2 . These results are in agreement with published reports that Acinetobacter harbors high prevalence of integron class 1 , lower class 2 and no class 3 (Koeleman et al., 2000; Ploy et al., 2000; Galleco and Towner, 2001; Gaur et al., 2006; $\mathrm{Xu}$ et al., 2008). The lack of integron class 3 may indicate its null role in antibiotic resistance. As mentioned above, the prevalence of class 1 integron, as compared to class 2 may imply that class 1 integron is more important in capturing resistant determinants. Alternatively, both systems acquire the same resistance genes but class 1 integrons may express these genes more efficiently. To determine this possibility, sequencing and cloning of resistance genes of the isolates containing class 1 or 2 integrons might be helpful. Comparison of antibiotic resistance patterns and their association with class 1 and 2 integrons confirms that both classes of integrons exhibit similar resistance patterns to the tested antibiotics (Table III). However, class 1 integron is more likely involved in emerging resistance to antibiotics.

Data in the present study show a statistical association between the presence of integrons and resistance to 6 antibiotics. Because we did not detect any association between resistance to other antibiotics and the presence of integrons, this can implicate the role of other resistance determinants (Gaur et al., 2006; Chen et al., 2010).

In conclusion, Acinetobacter expressed high resistance to most of the prescribed antibiotics. To reduce the resistance rate, comprehensive control measures along with determination of periodical antibiotic sensitivity pattern may alleviate the situation to an acceptable level. Colistin, imipenem and meropenem are the most effective agents against Acinetobacter. However, the clinical application of colistin is limited due to its inappropriate side effects.

\section{Acknowledgements \\ Deep thanks are due to prof. A. Alborzi for his invaluable help with provision of the laboratory facilities in Prof. Alborzi Clinical Microbiology Research Center. We are thankful to Hassan Khajehei, $\mathrm{PhD}$ for critical reading of the manuscript.}

\section{Literature}

Arakawa Y., M. Murakami, K. Suzuki, H. Ito, R. Wacharotayankun, S. Ohsuka, N. Kato and M. Ohta, M. 1995. A novel integron-like element carrying the metallo-b-lactamase gene blaIMP. Antimicrob. Agents. Chemother. 39: 1612-1615.

Bergogne-Berezin E. and K.J. Towner. 1996. Acinetobacter spp. as nosocomial pathogens: Microbiological, clinical \& epidemiological features. Clin. Microbiol. 8: 148-165.

Chen T.L., W.C. Chang, S.C. Kuo, Y.T. Lee, C.P. Chen, L.K. Siu, W.L. Cho and C.P. Fung. 2010. Contribution of a plasmid borne blaOXA-58 with its hybrid promoter provided by IS1006 and ISAba3-like to \{beta\}-lactam resistance in Acinetobacter genomic species 13TU. Antimicrob. Agents. Chemother. 54: 3107-3112.

Feizabadi M.M., B. fatollahzadeh, M. Taherikalani, M. Rasoolinejad, N. Sadeghiferd, M. Aligholi, S. Soroush and S. MohammadiYegane. 2008. Antimicrobial susceptibility patterns and distribution of bla ${ }_{\text {OXA }}$ genes among Acinetobacter spp. isolated from patients at Tehran hospitals. Jpn. J. Infect. Dis. 61: 274-278.

Galleco L. and K.J Towner. 2001. Carriage of class 1 integrons and antibiotic resistance in clinical isolates of Acinetobacter baumanni from Northern Spain. J. Med. Microbiol. 50: 71-77.

Gaur A., P. Prakash, S. Anupurba and T.M. Mahapatra. 2006 Possible role of integrase gene polymerase chain reaction as an epidemiological marker: study of multi-drug resistant Acinetobacter baumannii isolated from nosocomial infections. Int. J. Antimicrobial. Agents. 29: 446-450.

Gisneous J.M. and J. Rodriguez-Bano. 2002. Nosocomial bacteremia due to Acinetobacter baumannii: epidemiology, clinical features and treatment. Clin. Microbiol. Infects. 8: 687-693.

Gonzalez G., K. Sossa, H. Bello, M. Dominguez, S. Mella and R. Zemelman. 1998. Presence of integrons in isolates of different biotypes of Acinetobacter baumannii from Chilean hospitals. FEMs. Microbiol. lett. 161: 125-128.

Koeleman J.G., J. Stoof, M.W. Van Der bijl, C. M.VandenbrouckeGrauls and P.H. Savelkoul. 2000. Identification of epidemic strains of Acinetobacter baumannii by integrase gene PCR. J. Clin. Microbiol. 39: $8-13$.

Lévesque C., L. Piché, C. Larose and P.H. Roy. 1995. PCR mapping of integrons reveals several novel combinations of resistance genes. Antimicrob. Agents. Chemother. 39: 185-91.

Lewis J.R. and S.A. Lewis. 2004. Colistin interactions with mammalian urothelium. Am. J. physiol. Cell. physiol. 286: C913- C922. Neild B.S., A.J. Holmes, M.R. Gillings, G.D. Recchia, , B.C. Mabbutt, K.M. Nevalainen and H.W. Stokes. 2001. Recovery of new integron classes from environmental DNA. FEMS. Microbiol. Lett. 195: 59-65. Nordmann P. 2004. Acinetobacter baumannii, the nosocomial pathogen par excellence. Pathol. Biol. 52: 301-303. 
Perez F., A.M. Hujer, K.M. Hujer, B.K. Decker, P.N. Rather and R.A. Bonomo. 2007. Global challenge of multidrug-resistant Acinetobacter baumannii. Antimicrob. Agents. Chemother. 51: 3471-3484. Ploy M.C., F. Denis, P. Courvalin and T. Lambert. 2000. Molecular characterization of integrons in Acinetobacter baumannii: description of a hybrid class 2 integron. Antimicrob. Agents. Chemother. 44: 2684-2688

Radström P., O. Sköld, G. Swedberg, J. Flensburg, P.H. Roy and L. Sundström. 1994. Transposon Tn5090 of plasmid R751, which carries an integron, is related to $\mathrm{Tn} 7, \mathrm{Mu}$, and the retroelements. J. Bacteriol. 176: 3257-3268.

Reed M.D., R.C. Stern, M.A. O'Riordan and J.L. Blumer. 2001. The pharmacokinetics of colistin in patients with cystic fibrosis. J. Clin. Pharmacol. 41: 645-54.

Rowe-Magnus D. A. and D. Mazel. 1999. Resistance gene capture. Curr. Opin. Microbiol. 2: 481-486.
Seifert H., R. Baginsky, A. Schulze and G. Polverer. 1993. The distribution of Acinetobacter species in clinical culture materials. Zentralbl. Bakteriol. 279: 544-552.

Seward R.J. 1999. Detection of integrons in worldwide nosocomial isolates of Acinetobacter spp. Clin. Microbiol. Infect. 5: 308-318.

Towner K.J. 2009. Acinetobacter: an old friend, but a new enemy. J. Hosp. Infect. 73: 355-63.

Turton J.F., M.E. Kaufmann, J. Glover, J.M. Coelho, M. Warner, R. Pike and T.L. Pitt. 2005. Detection and typing of integrons in epidemic strains of Acinetobacter baumannii found in the United Kingdom. J. Clin. Microbiol. 43: 3074-3082.

Villegas M.V. and A.I. Hartstein. 2003. Acinetobacter outbreaks, 1977-2000. Infect. Control. Hosp. Epidemiol. 24: 284-95.

Xu X., F. Kong, X. Cheng, B. Yan X. Du, J. Gai, H.Ai, L. Shi and J. Iredell. 2008. Integron gene Cassettes in Acinetobacter spp. Strains from south China". Int. J. Antimicrobial. Agents. 32: 441-445. 